\title{
Positive Polynomials and Robust Stabilization with Fixed-Order Controllers*
}

\author{
Didier Henrion ${ }^{1,2}$, Michael ŠebeK ${ }^{3}$, Vladimír KuČera ${ }^{3}$
}

November 26, 2002

\begin{abstract}
Recent results on positive polynomials are used to obtain a convex inner approximation of the stability domain in the space of coefficients of a polynomial. An application to the design of fixed-order controllers robustly stabilizing a linear system subject to polytopic uncertainty is then proposed, based on LMI optimization. The key ingredient in the design procedure resides in the choice of a central polynomial, or desired nominal closed-loop characteristic polynomial. Several numerical examples illustrate the relevance of the approach.
\end{abstract}

\section{Keywords}

Polynomials, LMI, Robust Control, Structured Uncertainty, Fixed-Order Controllers.

\section{Introduction}

Most of the standard design procedures in robust control (LQG, $H_{2}$ or $H_{\infty}$ ) result in controllers of about the same order as the plant [Zhou, 1996]. Unfortunately, in several applications such as embedded control systems for the space and aeronautics industry, a controller of low order is a fundamental requirement. One must then resort to timeconsuming controller reduction techniques, not always with the guarantee that closed-loop

\footnotetext{
${ }^{1}$ Laboratoire d'Analyse et d'Architecture des Systèmes, Centre National de la Recherche Scientifique, 7 Avenue du Colonel Roche, 31077 Toulouse, cedex 4, France. Fax: +33 561336969 . E-mail: henrion@laas.fr

${ }^{2}$ Institute of Information Theory and Automation, Czech Academy of Sciences, Pod vodárenskou věží 4, 18208 Praha, Czech Republic.

${ }^{3}$ Center for Applied Cybernetics, Faculty of Electrical Engineering, Czech Technical University in Prague, Technická 2, 16627 Praha, Czech Republic.

*In memory of Filipe Andre Devy Vareta, who died tragically on May 30, 2002.
} 
performance is preserved. The main hindrance for the development of efficient design algorithms directly producing low order controllers resides in the fundamental algebraic property that the stability domain in the space of coefficients of a polynomial is a nonconvex set in general [Ackermann, 1993]. As a consequence, several very basic control problems such as multivariable static output feedback or simultaneous stabilization of three (or more) plants are still open. Indeed, there are no efficient (polynomial-time) algorithms to solve them [Blondel, 2000]. For more than thirty years now, researchers have been focusing on different approaches to address these difficult control problems, including computationally intensive global optimization, iterative heuristics without convergence guarantee, or sufficient stability conditions based on convex optimization. In this paper, we pursue the latter approach.

In Section 2 we propose a convex inner approximation of the non-convex stability domain in the space of polynomial coefficients. The derivation of such approximations with various shapes (polytopes, hyperspheres, ellipsoids) has always been a favorite topic among researchers, see [Ackermann, 1993] or [Bhattacharyya, 1995]. The stability domain proposed here is described by a linear matrix inequality (LMI, see [El Ghaoui, 1999]), so it is a convex object that can be unbounded. The LMI stability domain is parametrized by a given stable polynomial, referred to as the central polynomial for reasons that should become clear later on in the paper.

In Section 3 we show that the convex LMI approximation of the stability domain can be used to solve partially the difficult robust control problem of stabilizing a polytope of transfer functions with a controller of fixed (presumably low) order. Polytopic uncertainty is probably the most general way of capturing the structured uncertainty that may affect the system parameters. For example, it includes the well-known interval parametric uncertainty [Bhattacharyya, 1995]. Most results invoking polytopic systems are robust stability analysis results, see the introduction in [Henrion, 2001b] for a recent survey on existing techniques. A few design techniques have been proposed so far, see [Henrion, 2001a] and the references therein. In the present paper, we propose a new technique for robust stabilization of a polytopic system whose main strength is that the order of the controller is fixed from the outset.

Section 4 collects several numerical examples from the technical literature illustrating that our design technique may fulfill the requirement of simple and efficient low-order controller design algorithms. It is namely shown how the choice of the central polynomial parametrizing the LMI stability region is the key ingredient for a successful robust design. Roughly speaking, the central polynomial is the desired nominal closed-loop characteristic polynomial.

The ideas lying behind the derivation of the sufficient LMI stability condition are based partially on the theory of strictly positive real (SPR) functions. SPR functions have already been used for robust design in the control literature, see [Dorato, 2000] for a nice tutorial. Tools of functional analysis are invoked together with SPR functions to design robust controllers of arbitrarily high orders for discrete-time polytopic plants in [Anderson, 1990]. Later on, these results were combined with the Youla-Kučera parametrization of all stabilizing controllers to perform robust design on continuous-time systems affected by norm-bounded (ellipsoidal) uncertainty [Rantzer, 1994]. In [Abdallah, 1995] 
SPR functions are used to interpolate units in the space of stable rational functions, also resulting in controllers having potentially very high orders. More recently, in [Langer, 1999] the authors use SPR functions to optimize over the numerator of the system transfer function and satisfy frequency domain constraints on the sensitivity function, hence ensuring closed-loop robustness. To preserve convexity of the optimization problem, the denominator of the transfer function is first assigned via pole placement. Similarly, in [Wang, 2000] system poles are assigned and SPRness is invoked in a state-space framework to ensure closed-loop performance.

The contribution of our paper with respect to these references is in showing that the recently developed theory of positive polynomials (see [Nesterov, 2000, Parrilo, 2000, Lasserre, 2001] and the references therein) can be invoked together with properties of SPR functions to perform robust design in a purely polynomial or algebraic framework [Kučera, 1979], by opposition to rational, or state-space frameworks. Moreover, contrary to most of the design methods currently available, the order of the controller is fixed from the outset, generally resulting in a robust control law of low complexity. Finally, it is shown that the underlying LMI optimization problem has a special structure which may be exploited by fast, efficient semidefinite programming solvers such as SeDuMi [Sturm, 1999].

\section{Convex Inner Approximation of the Stability Do- main of a Polynomial}

In this section we show that the theory of positive polynomials can be invoked to obtain an inner approximation of the stability domain of a polynomial formulated as a linear inequality over the cone of positive semidefinite matrices, or LMI [El Ghaoui, 1999]. After submission of the first version of our paper, Bogdan Dumitrescu pointed out to us that similar results have been published independently in [Dumitrescu, 2002].

\subsection{LMI Approximation of the Stability Domain}

Let

$$
\mathcal{D}=\{s \in \mathbb{C}:\left[\begin{array}{l}
1 \\
s
\end{array}\right]^{\star} \underbrace{\left[\begin{array}{ll}
d_{11} & d_{12} \\
d_{12}^{\star} & d_{22}
\end{array}\right]}_{D}\left[\begin{array}{l}
1 \\
s
\end{array}\right]<0\}
$$

be a stability region in the complex plane, where the star denotes transpose conjugate and Hermitian matrix $D$ has one strictly positive eigenvalue and one strictly negative eigenvalue. Standard choices for $\mathcal{D}$ are the left half-plane $\left(d_{11}=0, d_{12}=1, d_{22}=0\right)$ and the unit disk $\left(d_{11}=-1, d_{12}=0, d_{22}=1\right)$. Other choices of scalars $d_{11}, d_{12}$ and $d_{22}$ correspond to arbitrary half-planes and disks. Let $\partial \mathcal{D}$ denote the one-dimensional boundary of $\mathcal{D}$, i.e. the set

$$
\partial \mathcal{D}=\left\{s \in \mathbb{C}: d_{11}+d_{12} s+d_{12}^{\star} s^{\star}+d_{22} s s^{\star}=0\right\}
$$


In the sequel we say that a polynomial is D-stable when its roots belong to $\mathcal{D}$. Similarly, we say that a rational function is D-strictly positive real (D-SPR) when its real part is positive when evaluated along $\partial \mathcal{D}$.

Let $\Pi_{i}$ denote a projection matrix of size $2 \times(n+1)$ with ones at entries $(1, i)$ and $(2, i+1)$ and zeros elsewhere. Let $D_{i j}=\Pi_{i}^{\star} D \Pi_{j}+\Pi_{j}^{\star} D \Pi_{i}$ for $i, j=1, \ldots, n$. Define the linear application

$$
D(Q)=\sum_{i=1}^{n} \sum_{j=1}^{n} D_{i j} q_{i j}
$$

mapping the space of Hermitian matrices $Q$ of size $n$ with entries $q_{i j}$ onto the space of Hermitian matrices of size $n+1$. Finally, let $H_{k}$ be linearly independent non-zero Hermitian matrices such that

$$
\text { trace } D_{i j} H_{k}=0, \quad k=0,1, \ldots, n \text {. }
$$

Finding matrices $H_{k}$ amounts to extracting a basis for the kernel of a matrix, an elementary operation of linear algebra. This will be clarified later with the help of a numerical example. The important thing to recall here is that the structure of matrices $H_{k}$ depends on stability matrix $D$ only.

Throughout the paper we consider polynomials $c(s)=c_{0}+c_{1} s+\cdots+c_{n} s^{n}$ and $d(s)=$ $d_{0}+d_{1} s+\cdots+d_{n} s^{n}$ of degree $n$, with real coefficient vectors

$$
c=\left[\begin{array}{llll}
c_{0} & c_{1} & \cdots & c_{n}
\end{array}\right], \quad d=\left[\begin{array}{llll}
d_{0} & d_{1} & \cdots & d_{n}
\end{array}\right] .
$$

Given an arbitrarily small positive scalar $\gamma$, we define the Hermitian matrix

$$
P(c)=c^{\star} d+d^{\star} c-2 \gamma d^{\star} d
$$

where the notation underlines a linear dependence of matrix $P$ in coefficients of polynomial $c(s)$. Finally, $A \succeq 0$ means that matrix $A$ is positive semidefinite.

We can now state the following fundamental result:

Theorem 1 Given a D-stable polynomial d(s) of degree $n$, polynomial c(s) is D-stable if there exists a matrix $X$ of size $n+1$ solving the primal LMI

$$
\text { trace } H_{k} P(c)=\text { trace } H_{k} X, \quad k=0,1, \ldots, n, \quad X=X^{\star} \succeq 0
$$

or equivalently, if there exists a matrix $Q$ of size $n$ solving the dual LMI

$$
P(c)+D(Q) \succeq 0, \quad Q=Q^{\star} .
$$

In the next section we shall prove that primal LMI (3) and dual LMI (4) are actually equivalent. The interpretation of Theorem 1 is as follows: as soon as polynomial $d(s)$ is fixed, we obtain a sufficient LMI condition for stability of polynomial $c(s)$. Equivalently, we obtain a convex inner approximation of the (generally non-convex) stability domain in the space of polynomial coefficients. In contrast with existing approximations (polytopes, hyperspheres, ellipsoids) the LMI stability domain has no specific shape and can be unbounded.

For reasons explained later in the paper, polynomial $d(s)$ will be referred to as the central polynomial. 


\subsection{Proof of Theorem 1}

First we need the following basic result:

Lemma 1 Polynomial $c(s)$ is D-stable if and only if there exists a D-stable polynomial $d(s)$ such that rational function $c(s) / d(s)$ is $D$-SPR.

Corollary 1 Given a D-stable polynomial $d(s)$, polynomial $c(s)$ is D-stable if rational function $c(s) / d(s)$ is $D$-SPR.

Proof: From the definition of a D-SPR rational function, $c(s) / d(s)$ D-SPR with $d(s)$ D-stable implies $c(s)$ D-stable. Conversely, if $c(s)$ is D-stable then the choice $d(s)=c(s)$ makes rational function $c(s) / d(s)=1$ obviously D-SPR.

The next step is then to formulate SPRness of a rational function as positivity of a polynomial. Relating SPRness to positivity is a well-known trick, see [Stipanović, 2000] for references. Let $\gamma$ denote some arbitrarily small positive scalar.

Lemma 2 The SPRness condition

$$
\operatorname{Re} \frac{c(s)}{d(s)}=\frac{1}{2}\left(\frac{c^{\star}(s)}{d^{\star}(s)}+\frac{c(s)}{d(s)}\right)=\frac{1}{2}\left(\frac{c^{\star}(s) d(s)+d^{\star}(s) c(s)}{d^{\star}(s) d(s)}\right) \geq \gamma
$$

is equivalent to the positivity (non-negativity) condition

$$
p(s)=c^{\star}(s) d(s)+d^{\star}(s) c(s)-2 \gamma d^{\star}(s) d(s) \geq 0
$$

for all $s \in \partial \mathcal{D}$.

Recalling the above definition of matrices $P(c)$ and $D(Q)$, polynomial positivity can be formulated as an LMI condition, as shown below:

Lemma 3 Polynomial $p(s)$ is positive along $\partial \mathcal{D}$ if and only if there exists a matrix $Q$ satisfying $\operatorname{LMI}(4)$.

Proof: First assume that there exists a matrix $Q$ satisfying LMI (4). Let

$$
\pi_{n}(s)=\left[\begin{array}{c}
1 \\
s \\
\vdots \\
s^{n}
\end{array}\right]
$$

and pre- and post-multiply LMI (4) by the above vector to obtain

$$
\pi_{n}^{\star}(s) P(c) \pi_{n}(s)+\pi_{n}^{\star}(s) D(Q) \pi_{n}(s) \geq 0
$$


where matrix $P(c)$ was defined in equation (2). From the structure of linear map $D(Q)$ one can check that the equality

$$
\pi_{n}^{\star}(s) D(Q) \pi_{n}(s)=\left(a+b s+b^{\star} s^{\star}+c s s^{\star}\right) \pi_{n-1}^{\star}(s) Q \pi_{n-1}(s)=0
$$

holds for all $s \in \partial \mathcal{D}$, so that

$$
p(s)=\pi_{n}^{\star}(s) P(c) \pi_{n}(s) \geq 0, \quad \forall s \in \partial \mathcal{D} .
$$

Conversely, assuming that polynomial $p(s)$ is nonnegative along $\partial \mathcal{D}$, we will show first that the set of all constant matrices $R$ such that

$$
\pi_{n}^{\star}(s) R \pi_{n}(s)=p(s), \quad \forall s \in \partial \mathcal{D}
$$

can be described as

$$
R=P(c)+D(Q)
$$

where $Q=Q^{\star}$ is an arbitrary matrix. Indeed, the whole set of polynomials of degree $n$ or less in $s$ and $s^{\star}$ vanishing along $\partial \mathcal{D}$ is described by the term $\pi_{n}^{\star}(s) D(q) \pi_{n}(s)$. To prove this, note that in equation (5) the vanishing scalar term $a+b s+b^{\star} s^{\star}+c s s^{\star}$ necessarily appears as a common factor in polynomial $\pi_{n}^{\star}(s) D(Q) \pi_{n}(s)$, while the remaining factor $\pi_{n-1}^{\star}(s) Q \pi_{n-1}(s)$ describes the whole set of polynomials of degree $n-1$ or less. The last step in the proof then consists in showing that there always exists a matrix $Q=Q^{\star}$ such that $R \succeq 0$ as soon as $p(s) \geq 0$ along $\partial \mathcal{D}$. This follows from the existence of a decomposition of nonnegative polynomial $p(s)$ as a sum of squares, see [Nesterov, 2000, Parrilo, 2000, Lasserre, 2001] for recent overviews.

The last step in proving Theorem 1 consists in establishing the equivalence between primal LMI (3) and dual LMI (4):

Lemma 4 Primal LMI (3) is feasible if and only if dual LMI (4) is feasible.

Proof: It follows by application of Finsler's Lemma, see e.g. [Skelton, 1998]. LMI (3) is just a projection of LMI (4) onto the kernel of linear map $D(Q)$. The projection results in the elimination of matrix variable $Q$.

The advantage of formulating the sufficient condition of stability for a polynomial as an LMI arising from positivity conditions on polynomials is that these convex optimization problems have a special structure (Toeplitz structure for discrete-time polynomials, or Hankel structure for continuous-time polynomials) which may be exploited to design efficient algorithms, see [Nesterov, 2000, Genin, 2002] or [Alkire, 2001]. Numerical properties of these algorithms must be however studied in further detail, since it is known for example that positive definite Hankel matrices are exponentially ill-conditioned. Such considerations remain however out of the scope of this paper. 


\subsection{Shape of the LMI Stability Domain for a Second Degree Discrete-Time Polynomial}

In this paragraph, we characterize the LMI stability domain of Theorem 1 in the special case of a second degree monic discrete-time polynomial

$$
c(z)=c_{0}+c_{1} z+z^{2}
$$

when the central polynomial is set to

$$
d(z)=z^{2} .
$$

The motivation behind this choice is twofold. First we can obtain an analytical expression of the approximate LMI stability domain. Second we know that the exact stability domain is the convex interior of a triangle with vertices $-1+z+z^{2}, 1-2 z+z^{2}$ and $1+2 z+z^{2}$ [Ackermann, 1993]. Thus we believe that the following study provides additional insight into the structure of the LMI optimization problems arising from positivity conditions on polynomials.

Stability region $\mathcal{D}$ is the interior of the unit disk, i.e.

$$
D=\left[\begin{array}{cc}
-1 & 0 \\
0 & 1
\end{array}\right]
$$

and matrices $H_{k}$ for $k=0,1,2$ introduced in Section 2.1 are obtained as follows. Let vec denote the column vector obtained by stacking the columns of the upper triangular part of a matrix. Then, equality constraints (1) can be written as

$$
\left[\begin{array}{lll}
\operatorname{vec}\left(D_{11}\right) & \operatorname{vec}\left(D_{12}\right) & \operatorname{vec}\left(D_{22}\right)
\end{array}\right]^{\star}\left[\begin{array}{lll}
\operatorname{vec}\left(H_{0}\right) & \operatorname{vec}\left(H_{1}\right) & \operatorname{vec}\left(H_{2}\right)
\end{array}\right]=0
$$

i.e. Hermitian matrices $H_{k}$ are obtained by extracting a basis for the right null-space of a matrix whose rows are built from Hermitian matrices $D_{i j}$. For the above choice of stability matrix $D$, we have

$$
D_{11}=\left[\begin{array}{ccc}
-2 & 0 & 0 \\
0 & 2 & 0 \\
0 & 0 & 0
\end{array}\right], \quad D_{12}=\left[\begin{array}{ccc}
0 & -1 & 0 \\
-1 & 0 & 1 \\
0 & 1 & 0
\end{array}\right], \quad D_{22}=\left[\begin{array}{ccc}
0 & 0 & 0 \\
0 & -2 & 0 \\
0 & 0 & 2
\end{array}\right] \text {. }
$$

A possible candidate for the null-space basis is as follows:

$$
\left[\begin{array}{c|cc|ccc}
-2 & 0 & 2 & 0 & 0 & 0 \\
0 & -1 & 0 & 0 & 1 & 0 \\
0 & 0 & -2 & 0 & 0 & 2
\end{array}\right]\left[\begin{array}{ccc}
0 & 0 & 1 \\
0 & 1 & 0 \\
0 & 0 & 1 \\
\hline 1 & 0 & 0 \\
0 & 1 & 0 \\
0 & 0 & 1
\end{array}\right]=0
$$

so a possible choice of matrices $H_{k}$ is:

$$
H_{0}=\left[\begin{array}{lll}
0 & 0 & 1 \\
0 & 0 & 0 \\
1 & 0 & 0
\end{array}\right], \quad H_{1}=\left[\begin{array}{lll}
0 & 1 & 0 \\
1 & 0 & 1 \\
0 & 1 & 0
\end{array}\right], \quad H_{2}=\left[\begin{array}{lll}
1 & 0 & 0 \\
0 & 1 & 0 \\
0 & 0 & 1
\end{array}\right] .
$$


Note that matrices $H_{k}$ are Toeplitz, which is always the case for discrete-time polynomials.

In virtue of Theorem 1 , the approximate stability domain is the set of coefficients $c_{0}, c_{1}$ for which LMI (3) is feasible, i.e. for which there exists a matrix

$$
X=X^{\star}=\left[\begin{array}{lll}
x_{00} & x_{01} & x_{02} \\
x_{10} & x_{11} & x_{12} \\
x_{20} & x_{21} & x_{22}
\end{array}\right] \succeq 0
$$

such that

$$
\begin{aligned}
x_{00}+x_{11}+x_{22} & =2-2 \gamma \\
x_{10}+x_{01}+x_{21}+x_{22} & =c_{1} \\
x_{20}+x_{02} & =c_{0} .
\end{aligned}
$$

There are more unknowns than constraints in this problem, so it is more convenient to work on the dual problem. Recalling standard results on semidefinite programming duality [Vandenberghe, 1996] and defining mat $(x)$ as the square matrix whose columns are stacked in vector $x$, there is no vector $x$ such that

$$
A x=b, \quad X=X^{\star}=\operatorname{mat}(x) \succeq 0
$$

if and only if there exists a Farkas dual vector $y$ such that

$$
b^{\star} y<0, \quad Y=Y^{\star}=\operatorname{mat}\left(A^{\star} y\right) \succeq 0,
$$

provided there exists at least one vector $y$ such that mat $\left(A^{\star} y\right) \succ 0$.

In our case, linear system (6) can be written as $A x=b$ with $x=\operatorname{mat}(X)$ and

$$
A=\left[\begin{array}{lllllllll}
1 & 0 & 0 & 0 & 1 & 0 & 0 & 0 & 1 \\
0 & 1 & 0 & 1 & 0 & 1 & 0 & 1 & 0 \\
0 & 0 & 1 & 0 & 0 & 0 & 1 & 0 & 0
\end{array}\right], \quad b=\left[\begin{array}{c}
1 \\
c_{1} \\
c_{0}
\end{array}\right]
$$

where we have normalized to one the first equality constraint. This is done without loss of generality since $\gamma$ is a small positive scalar and matrix $H_{2}$ can be scaled arbitrarily. Infeasibility of this problem is then equivalent to existence of a vector $y=\left[\begin{array}{lll}y_{0} & y_{1} & y_{2}\end{array}\right]^{\star}$ such that $y_{0}+c_{1} y_{1}+c_{0} y_{2}<0$ and

$$
Y=\operatorname{mat}\left(A^{\star} y\right)=\left[\begin{array}{lll}
y_{0} & y_{1} & y_{2} \\
y_{1} & y_{0} & y_{1} \\
y_{2} & y_{1} & y_{0}
\end{array}\right] \succeq 0
$$

Indeed, the eigenvalues of symmetric Toeplitz matrix $Y$ are equal to $y_{0}-y_{2}$ and $\left(2 y_{0}+\right.$ $\left.y_{2} \pm \sqrt{y_{2}^{2}+8 y_{1}^{2}}\right) / 2$ so it is always possible to find a vector $y$ such that $Y \succ 0$. Matrix $Y$ is positive semidefinite if and only if $y_{2} \leq y_{0}$ and $y_{1}^{2} \leq\left(y_{0}+y_{2}\right) y_{0} / 2$, i.e. if and only if $y_{1}$ and $y_{2}$ belong to the interior of a bounded parabola scaled by $y_{0}$.

The corresponding values of $c_{0}$ and $c_{1}$ belong to the interior of the envelope generated by the curve

$$
\left(2 \lambda_{2}-1\right) c_{0}+\left(2 \lambda_{1}-1\right) \sqrt{\lambda_{2}} c_{1}+1>0, \quad 0 \leq \lambda_{1}, \lambda_{2} \leq 1
$$


The implicit equation of the envelope is given by

$$
8 c_{0}^{2}-8 c_{0}+c_{1}=0, \quad\left(2 c_{0}-1\right)^{2}+\left(\frac{\sqrt{2}}{2} c_{1}\right)^{2}=1
$$

which is an ellipse with axes parallel to the coordinate axes. The LMI stability region is then the union of the interior of the ellipse with the interior of the triangle delimited by the two lines

$$
c_{0} \pm c_{1}+1=0
$$

tangent to the ellipse, with vertices $[-1,0],[1 / 3,4 / 3]$ and $[1 / 3,-4 / 3]$, see Figure 1 .

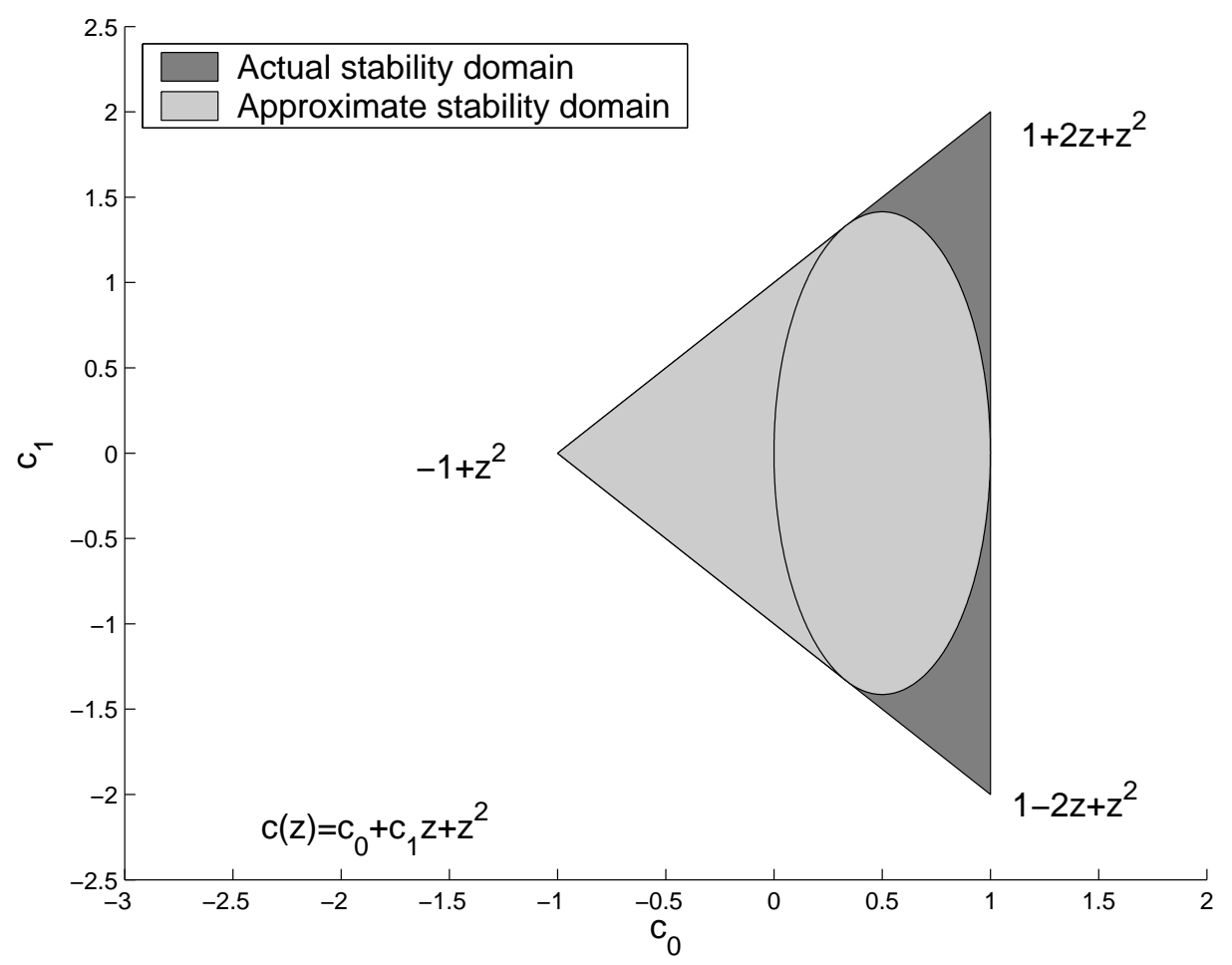

Figure 1: Actual stability domain (triangle) and approximate LMI stability domain for a discrete-time polynomial of degree two.

Of course, for different values of stable central polynomial $d(z)$ we obtain different shapes for the LMI stability domain. Using Lemma 1 it can be shown the whole actual stability domain (i.e. the whole triangle) is covered when $d(z)$ covers the whole set of stable polynomials.

\section{Robust Stabilization of a Polytope of Polynomials with a Fixed-Order Controller}

Now we use the convex inner approximation of the stability domain proposed in Section 2 to address the difficult problem of robust stabilization of a polytope of polynomials with 
a controller of fixed order. As pointed out in the introduction, due to the non-convexity of the stability domain in the space of closed-loop characteristic polynomial coefficients, there are very few fixed-order controller design algorithms available. In the sequel, we describe an algorithm that has the merit of being simple and very easy to implement. However, as it is based on a sufficient stability condition, when the algorithm fails, then we cannot conclude about robust stabilization of the polytopic system.

The central feature of the algorithm is in the choice of the central polynomial.

\subsection{Problem Statement}

Consider the standard negative feedback configuration of Figure 2 where $a(s), b(s)$ are

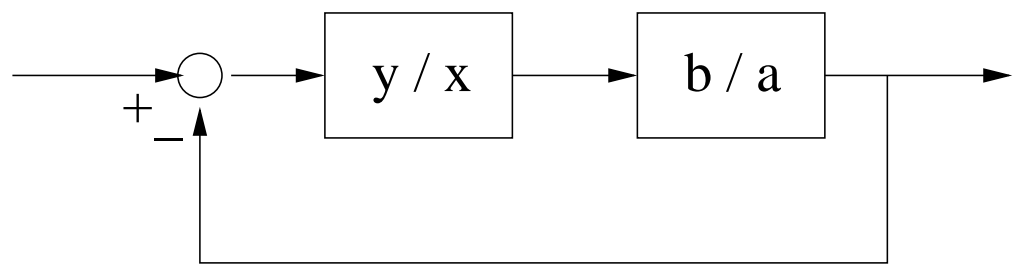

Figure 2: Feedback configuration.

polynomials of degree $n$ describing a scalar plant and $x(s), y(s)$ are controller polynomials of degree $m$ that must be found. We assume that the plant is subject to structured parametric uncertainty: transfer function $b(s) / a(s)$ belongs to a polytope with $N$ given vertices $b^{1}(s) / a^{1}(s), \ldots, b^{N}(s) / a^{N}(s)$.

Robust stabilization of the uncertain plant is then equivalent to the existence of controller polynomials $x(s)$ and $y(s)$ such that the whole polytope of closed-loop characteristic polynomials

$$
c(s)=a(s) x(s)+b(s) y(s)
$$

of degree $m+n$ with vertices $c^{i}(s)=a^{i}(s) x(s)+b^{i}(s) y(s)$ for $i=1, \ldots, N$. Note that this problem is more general than the simultaneous stabilization problem, where only the $N$ polytope vertices $c^{i}(s)$ must be stabilized.

\subsection{Sufficient LMI Condition For Robust Stabilization}

Denoting by $c^{i}$ the coefficient vector of vertex characteristic polynomial $c^{i}(s)$, and identifiying increasing powers of the indeterminate in the polynomial equation $c^{i}(s)=a^{i}(s) x(s)+$ 
$b^{i}(s) y(s)$, we obtain

$$
\begin{aligned}
& c^{i}=\left[\begin{array}{llll}
c_{0}^{i} & c_{1}^{i} & \cdots & c_{n+m}^{i}
\end{array}\right]= \\
& {\left[\begin{array}{llll|llll}
x_{0} & x_{1} & \cdots & x_{m} \mid y_{0} & y_{1} & \cdots & y_{m}
\end{array}\right]\left[\begin{array}{ccccccc}
a_{0} & a_{1} & \cdots & a_{n} & & & \\
& a_{0} & a_{1} & \cdots & a_{n} & & \\
& & \ddots & & & \ddots & \\
& & & a_{0} & a_{1} & \cdots & a_{n} \\
& & & &
\end{array}\right] .}
\end{aligned}
$$

As in equation (2) we define a matrix $P\left(c^{i}\right)$, that we denote here by $P^{i}(x, y)$ to underline the linear dependence in controller coefficients $x$ and $y$ :

$$
P^{i}(x, y)=\left(c^{i}\right)^{\star} d+d^{\star} c^{i}-2 \gamma d^{\star} d .
$$

In the above equation, $d$ is the coefficient vector of the given central polynomial $d(s)$, and $\gamma$ is an arbitrarily small positive scalar.

Based on Theorem 1, we can now state the main result of this paper:

Theorem 2 Let $a^{i}(s)$ and $b^{i}(s)$ for $i=1, \ldots, N$ be respectively denominator and numerator vertex polynomials of degree $n$ of a polytopic plant. Let $d(s)$ be a D-stable polynomial of degree $n+m$. If there exist matrices $X^{i}$ satisfying the primal LMI

$$
\operatorname{trace} H_{k} P^{i}(x, y)=\operatorname{trace} H_{k} X^{i}, \quad k=0,1, \ldots, n, \quad X^{i}=\left(X^{i}\right)^{\star} \succeq 0, \quad i=1, \ldots, N
$$

or equivalently, if there exist matrices $Q^{i}$ solving the dual LMI

$$
P^{i}(x, y)+D\left(Q^{i}\right) \succeq 0, \quad Q^{i}=\left(Q^{i}\right)^{\star}, \quad i=1, \ldots, N
$$

then the controller with denominator and numerator polynomials $x(s)$ and $y(s)$ robustly D-stabilizes the polytopic plant.

Proof Recalling Theorem 1, given a D-stable central polynomial $d(s)$, a sufficient condition for D-stability of polynomial $c(s)=a(s) x(s)+b(s) y(s)$ is the feasibility of LMI (3) or (4) which are linear in the coefficients of $c(s)$, thus linear in the coefficients of $x(s)$ and $y(s)$. Since the coefficients of polytopic system polynomials $a^{i}(s)$ and $b^{i}(s)$ also appear linearly, it is necessary and sufficient that LMI (7) or (8) hold at each vertex $i=1, \ldots, N$.

Of course, the LMI condition in Theorem 2 is only sufficient for robust stabilization since we assume that a central polynomial $d(s)$ is given as input data. It is the designer's task to find what is the appropriate choice for central polynomial $d(s)$. It is of upmost importance that $d(s)$ be a D-stable polynomial, i.e. all its roots must lie within the stability region $\mathcal{D}$ chosen for design. Generally, sensible choices for $d(s)$ are either 
- the nominal closed-loop characteristic polynomial, or

- any vertex characteristic polynomial $d(s)=c^{i}(s)=a^{i}(s) x(s)+b^{i}(s) y(s)$ computed with any standard design algorithm [Kučera, 1979], or

- any polynomial $d(s)$ whose zeros are sufficiently close to the closed-loop characteristic polynomial zeros.

Roughly speaking, the set of zeros of $d(s)$ will somehow match the expected closed-loop spectrum, which motivated the name "central polynomial" for $d(s)$. The choice of $d(s)$ must be consistent with the achievable process dynamics. The reader will find more on the choice of $d(s)$ in the next section on numerical examples.

Since the design LMI (in primal or dual form) of Theorem 2 is linear in the controller parameters, we can enforce structural constraints on the controller. For example, we can enforce the controller to be a PI controller, as soon as $m=1$ and $x_{0}=0, x_{1}=1$.

Because there is no criterion to optimize in the design LMI, we will minimize the Euclidean norm of the vector of controller parameters (this can always be formulated as an additional second-order cone constraint), provided the controller denominator is restricted to be monic, i.e. $x_{m}=1$.

\section{Numerical Examples}

The numerical examples were treated with the help of Matlab $6.1^{1}$ running under SunOS release 5.8 on a SunBlade 100 workstation. The value of $\gamma$ was set to $10^{-3}$. Operations on polynomials were performed with the Polynomial Toolbox 2.5 [Polyx Ltd., 2001]. The semidefinite programming problems were solved with SeDuMi 1.05 [Sturm, 1999].

\subsection{F4E aircraft}

We consider a model of the longitudinal motion of an F4E fighter aircraft [Ackermann, 1993, $\S 1.4]$. The input is the elevator position, the output is the pitch rate, and the system is linearized around four representative flight conditions in the Mach-altitude envelope:

$$
\begin{array}{lrll}
\text { Mach 0.5 } & 5000 \mathrm{ft} & a^{1}(s)=-52.75+22.00 s+15.84 s^{2}+s^{3} & b^{1}(s)=-163.8-185.4 s \\
\text { Mach 0.85 } & 5000 \mathrm{ft} & a^{2}(s)=-122.5+34.93 s+17.12 s^{2}+s^{3} & b^{2}(s)=-789.1-507.8 s \\
\text { Mach 0.9 } & 35000 \mathrm{ft} & a^{3}(s)=-14.64+17.51 s+15.33 s^{2}+s^{3} & b^{3}(s)=-101.8-158.3 s \\
\text { Mach 1.5 } & 35000 \mathrm{ft} & a^{4}(s)=269.1+43.60 s+15.74 s^{2}+s^{3} & b^{4}(s)=-251.4-304.2 s .
\end{array}
$$

We are seeking a static output feedback controller simultaneously stabilizing the four plants with a stability margin of 0.5 , i.e. we set

\footnotetext{
${ }^{1}$ Matlab is a trademark of The MathWorks, Inc.
}

$$
D=\left[\begin{array}{ll}
1 & 1 \\
1 & 0
\end{array}\right]
$$




\begin{tabular}{l|l}
$i$ & roots of $c^{i}(s)$ \\
\hline 1 & $-0.5118,-7.665 \pm j 10.80$ \\
2 & $-1.234,-7.943 \pm j 19.85$ \\
3 & $-0.5000,-7.413 \pm j 9.636$ \\
4 & $-1.717,-7.012 \pm j 15.33$
\end{tabular}

Table 1: F4E aircraft. Closed-loop poles.

as the stability matrix.

It is easy to see that the first plant can be D-stabilized with the controller polynomials $x_{1}(s)=1$ and $y_{1}(s)=-1$. The resulting characteristic polynomial

$$
d(s)=a^{1}(s) x^{1}(s)+b^{1}(s) y^{1}(s)=111.1+207.4 s+15.84 s^{2}+s^{3}
$$

has roots -0.5588 and $-7.6410 \pm j 11.85$ ensuring the stability margin. With the above four vertex plants $a^{i}(s), b^{i}(s)$ and the above central polynomial $d(s)$, we solved the LMI of Theorem 2 after less than 1 second of CPU time to obtain the controller

$$
\frac{y(s)}{x(s)}=-0.8698 \text {. }
$$

One can check that the above controller simultaneously D-stabilizes the four plants with the required stability margin. The roots of characteristic polynomials $c^{i}(s)=a^{i}(s) x(s)+$ $b^{i}(s) y(s)$ are given in Table 1. Actually the above controller does not only D-stabilize simultaneously the four plants but also any plant within the convex hull of these four vertices, see the edges of the robust root locus on Figure 3. This is a by-product of the approach.

\subsection{Robot}

We consider the problem of designing a robust controller for the approximate ARMAX model of a PUMA 762 robotic disk grinding process [Tong, 1994]. From the results of identification and because of the nonlinearity of the robot, the coefficients of the numerator of the plant transfer function change for different positions of the robot arm. We consider variations of up to $20 \%$ around the nominal value of the parameters. The fourth-order discrete-time model is given by

$$
\frac{b\left(z^{-1}, q\right)}{a\left(z^{-1}, q\right)}=\frac{\left(0.0257+q_{1}\right)+\left(-0.0764+q_{2}\right) z^{-1}+\left(-0.1619+q_{3}\right) z^{-2}+\left(-0.1688+q_{4}\right) z^{-3}}{1-1.914 z^{-1}+1.779 z^{-2}-1.0265 z^{-3}+0.2508 z^{-4}}
$$

where

$$
\left|q_{1}\right| \leq 0.00514,\left|q_{2}\right| \leq 0.01528,\left|q_{3}\right| \leq 0.03238,\left|q_{4}\right| \leq 0.03376
$$

are uncertain system parameters. The resulting polytopic system is then a four-dimensional box with $2^{4}=16$ vertices. The characteristic polynomial of the closed-loop system is given by

$$
c(z, q)=z^{12}\left[\left(1-z^{-1}\right) a\left(z^{-1}, q\right) x\left(z^{-1}\right)+z^{-5} b\left(z^{-1}, q\right) y\left(z^{-1}\right)\right]
$$




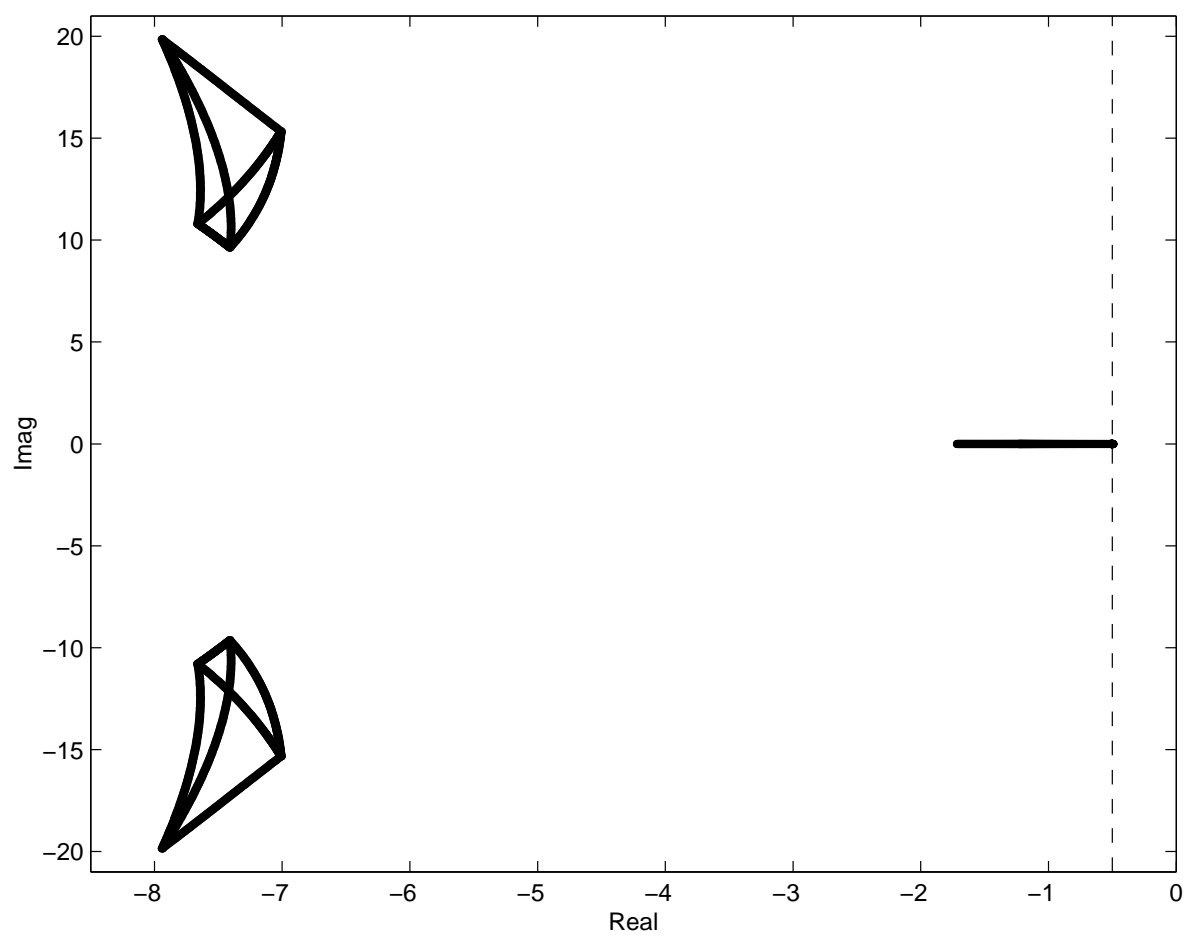

Figure 3: F4E aircraft. Edges of robust root locus.

where the term $1-z^{-1}$ is introduced in the controller denominator to maintain the steady state error to zero when parameters are changed. With the stable central polynomial

$$
d(z)=z^{19}
$$

and the discrete-time stability matrix

$$
D=\left[\begin{array}{cc}
-1 & 0 \\
0 & 1
\end{array}\right]
$$

the LMI of Theorem 2 is solved after 14 second of CPU time, resulting in the seventh-order robust controller

$\frac{y\left(z^{-1}\right)}{x\left(z^{-1}\right)}=\frac{-0.2863+0.2928 z^{-1}+0.0221 z^{-2}-0.1558 z^{-3}+0.0809 z^{-4}+0.1420 z^{-5}-0.1254 z^{-6}+0.0281 z^{-7}}{1+1.1590 z^{-1}+0.9428 z^{-2}+0.4996 z^{-3}+0.3044 z^{-4}+0.4881 z^{-5}+0.4003 z^{-6}+0.3660 z^{-7}}$.

The robust root locus, obtained by taking 1000 random plants within the uncertainty polytope, is represented on Figure 4.

\subsection{Gain margin optimization}

Finally we show how the gain margin can be improved by proper choice of the central polynomial. 


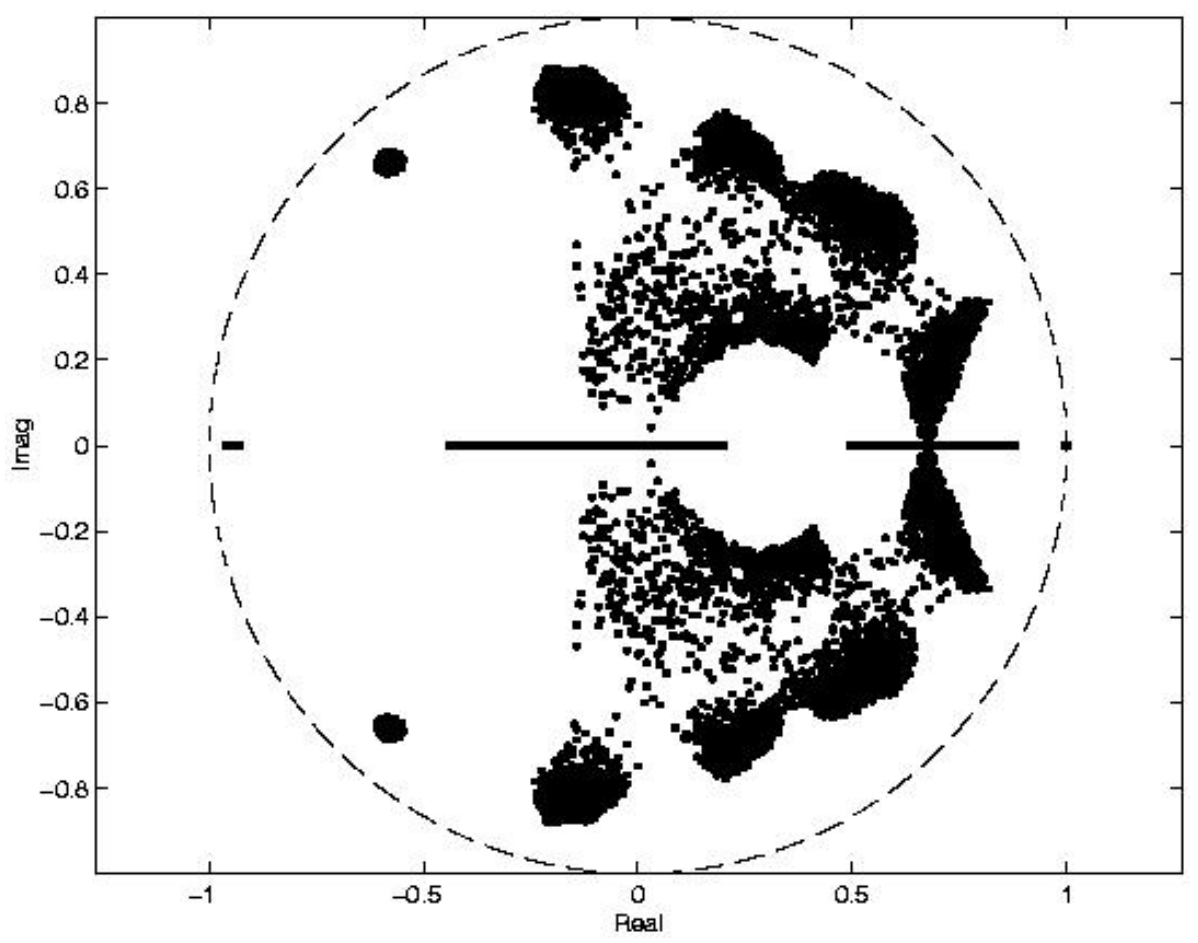

Figure 4: Robot. Robust root locus.

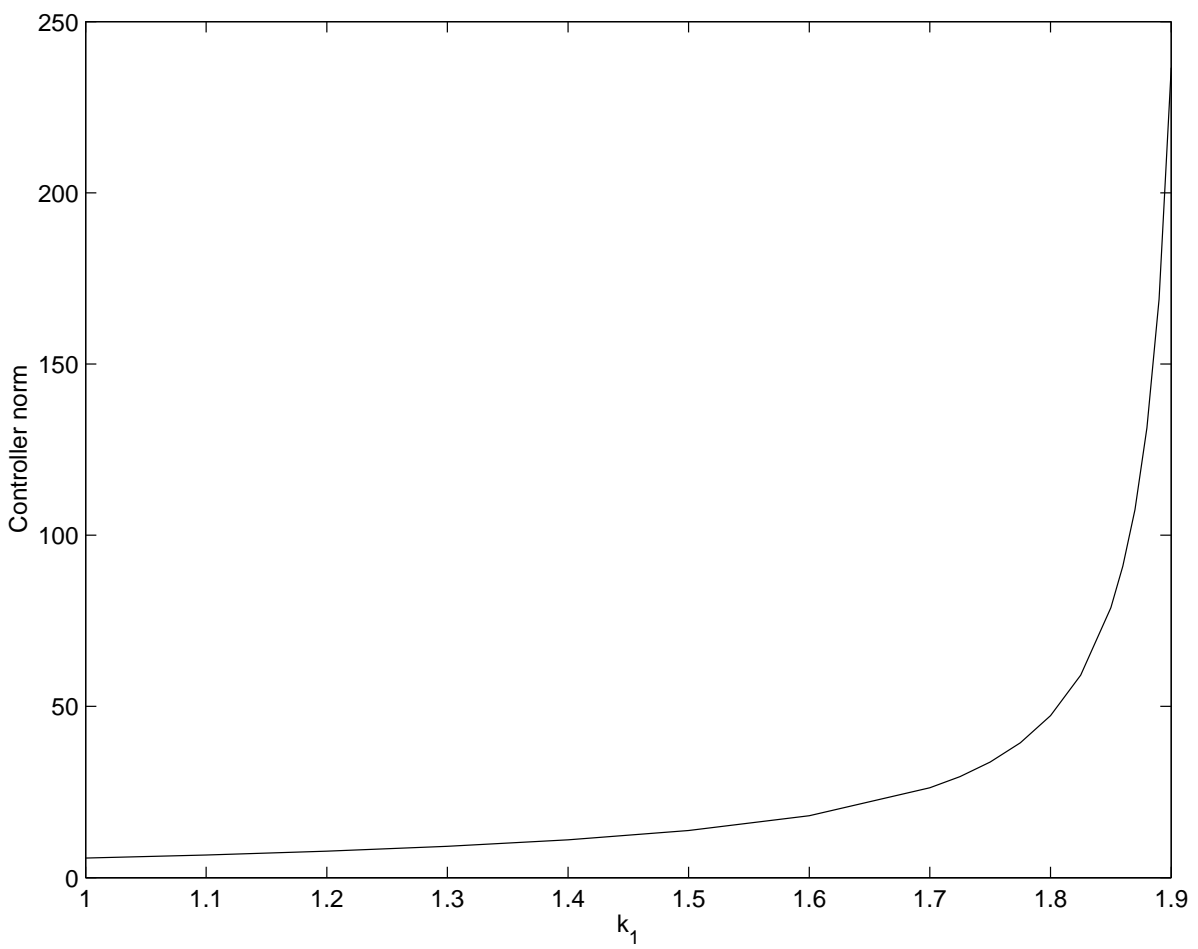

Figure 5: Norm of the first order controller as a function of upper gain $k_{1}$ with the central polynomial $c(s)=(s+1)^{3}$. 


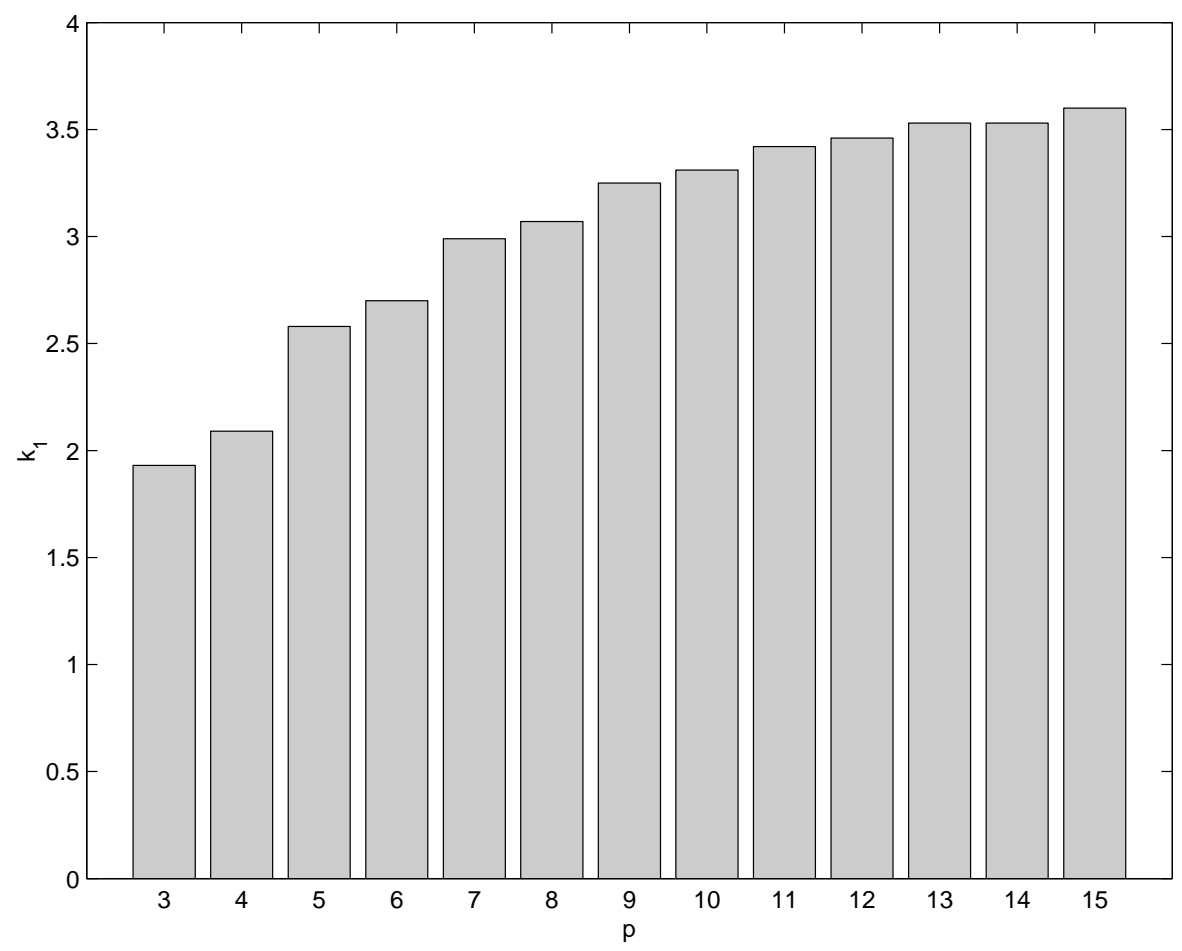

Figure 6: Maximum value of upper gain $k_{1}$ for which a first order robust controller is found with the central polynomial $d(s)=(s+1)^{p}$.

We consider as in [Doyle, 1992, §11.3] the problem of robustly stabilizing the plant

$$
\frac{b(s, q)}{a(s, q)}=\frac{q(s-1)}{(s+1)(s-2)}
$$

for all real gains $q$ in the interval $\left[1, k_{1}\right]$. The uncertain plant polytope is therefore made of 2 vertices only. In [Doyle, 1992] it is shown that a robustly stabilizing controller (of arbitrarily high order) exists if and only if $k_{1}<4$. The design method proposed there is based on coprime factorization and $H_{\infty}$ model matching. It is solved with the help of Nevanlinna-Pick interpolation, which has the drawback of producing high-order controllers. In [Doyle, 1992] a controller of eighth order is computed for $k_{1}=3.5$.

From the Hurwitz stability criterion there is no static controller stabilizing the plant, so we try the central polynomial $d(s)=(s+1)^{2+m}$ with stability matrix

$$
D=\left[\begin{array}{ll}
0 & 1 \\
1 & 0
\end{array}\right]
$$

in order to seek a continuous-time stabilizing controller of order $m$. When $m=1$ we represent in Figure 5 the Euclidean norm of the first order controller obtained by solving the LMI of Theorem 2 as a function of $k_{1}$. Recall that the LMI is solved by minimizing the Euclidean norm of the controller vector over all candidate controllers in the convex parametrization. In Figure 6 we reported as a function of degree $p=2+m$ the maximum value of $k_{1}$ for which a robust controller is found. For values of $p$ greater than 15 solver SeDuMi generally fails for numerical reasons and does not return any useful result. 
In the sequel we describe a heuristic to improve the gain margin with low-order controllers. Let $k_{1}=2$. We know from the results above that $d(s)=(s+1)^{3}$ is not a suitable choice of central polynomial for this value of $k_{1}$. It may mean that the poles of central polynomial $d(s)$ are not correctly chosen. So we try to move one pole nearest to the imaginary axis, just as in $d(s)=(s+1)^{2}(s+0.1)$ but the LMI is infeasible as well. We try the opposite direction, i.e. $d(s)=(s+1)^{2}(s+10)$ and the LMI solver now successfully returns a robustly stabilizing first-order controller

$$
\frac{y(s)}{x(s)}=\frac{254.9+348.1 s}{-327.9+s} .
$$

With this choice of central polynomial $d(s)$ the design LMI is feasible for values up to $k_{1}=2.38$, so we have improved the gain margin without increasing the controller order. Pursuing this idea and trying to move poles of $d(s)$, we have been able to stabilize robustly the system for $k_{1}=2.59$ and $d(s)=(s+0.5)(s+1)(s+100)$ with the first-order controller

$$
\frac{y(s)}{x(s)}=\frac{1292+1773 s}{-1731+s} .
$$

This gain margin may perhaps be improved with further attempts.

Proceeding similarly with higher order controllers, we have been able to stabilize robustly the plant for $k_{1}=3.5$ with the third-order controller

$$
\frac{y(s)}{x(s)}=\frac{240.8+755.5 s+871.7 s^{2}+420.1 s^{3}}{-423.5-739.8 s-409.5 s^{2}+s^{3}}
$$

with the choice

$$
d(s)=(s+0.5)^{3}(s+10)(s+100)
$$

as a central polynomial. Following this procedure, we may think about designing a heuristic to select poles of $d(s)$ and improve the gain margin without increasing the controller order.

It is however necessary to underline that the gain margin optimization problem as stated here has little practical relevance. Indeed, one can show that it is not possible to obtain a satisfactory phase margin for this system [Åström, 2000]. Generally speaking, closed-loop robustness cannot be ensured only via pole placement, and other criteria must be taken into account, such as the norm of sensitivity functions [Langer, 1999].

\section{Conclusion}

With the help of the theory of positive polynomials, we have described a very simple algorithm to design a fixed-order robust controller for a linear system affected by polytopic parametric uncertainty. It is based on a convex LMI approximation of the stability domain. The key ingredient of the design procedure resides in the choice of a central polynomial, which is the desired nominal closed-loop characteristic polynomial. Numerical experiments show that for most of the treated examples, an intuitive choice of a central polynomial often produces satisfying results. 
Contrary to most of the robust design algorithms based on SPR functions or the YoulaKučera parametrization [Rantzer, 1994, Dorato, 2000], the order of the controller is fixed from the very outset. Contrary to the parametric approach to robust design, where an ellipsoidal or polytopic approximation of the stability region must be known to assign closedloop polynomial coefficients [Ackermann, 1993, Bhattacharyya, 1995], the sole choice of the central polynomial ensures convexity of the problem. The convex approximation of the stability is then a possibly unbounded LMI region around the central polynomial.

In the special case of interval polynomials (when polynomial coefficients are assumed to belong to independent intervals of given lengths) we can think of a conceptual iterative algorithm based on successive improvements of the central polynomial. The starting point would be a nominal design performed with any standard method such as e.g. pole placement. The level of uncertainty (here the length of the coefficient intervals) would then be gradually increased, and the nominal closed-loop characteristic polynomial achieved by the robust LMI design method at iteration $k-1$ would be used as an input central polynomial at iteration $k$. The LMI design problem remains feasible provided the uncertainty level is increased gradually. The iterative procedure is stopped until no further improvement is possible.

Besides this, one key feature of the approach is that the special structure of the LMI formulation allows to apply specialized algorithms with low computational complexity. Generally speaking, it is believed that convex LMI optimization problems arising from properties of positive polynomials can be solved more efficiently than general LMI problems, see the recent results and discussions in [Nesterov, 2000, Genin, 2002] and also [Alkire, 2001]. Numerical conditionning improvement and the use of alternative polynomial bases such as Chebyshev polynomials are however still subjects of further investigation.

Another appealing characteristic of this design approach is that it applies indifferently to continuous-time or discrete-time scalar or multivariable systems. Although not studied in this paper, multi-input multi-output systems can indeed be handled exactly the same way as scalar systems, since properties of positive polynomial matrices entirely mimic properties of positive scalar polynomials [Genin, 2002]. More general stability regions, such as LMI regions [Chilali, 1996] or quadratic matrix inequality regions [Peaucelle, 2000] can also be handled without too much difficulty, based on results published in [Henrion, 2001c].

Other uncertainty models can also be considered, such as ellipsoidal uncertainty arising naturally from covariance information during the identification process [Braatz, 1998], or coprime factor additive or multiplicative uncertainty as considered in [Vidyasagar, 1985]. In principle, it should be possible to handle various uncertainty models via the concept of robust semidefinite programming introduced recently in [Ben-Tal, 2000].

\section{Acknowledgment}

This work was supported by the Grant Agency of the Czech Republic under Project No. 102/02/0709, by the Barrande Project No. 03080XJ/2001-031-1 and by the NATO 
Collaborative Linkage Grant No. PST.CLG.978481.

\section{References}

[Abdallah, 1995] C. Abdallah, P. Dorato, F. Pérez and D. Docampo. Controller Synthesis for a Class of Interval Plants. Automatica, Vol. 31, No. 2, pp. 341-343, 1995.

[Ackermann, 1993] J. Ackermann. Robust Control. Systems with Uncertain Physical Parameters. Springer Verlag, Berlin, 1993.

[Alkire, 2001] B. Alkire and L. Vandenberghe. Interior-Point Methods for Magnitude Filter Design. Proceedings of the IEEE International Conference on Acoustics, Speech and Signal Processing, Salt Lake City, Itah, May 2001.

[Anderson, 1990] B. D. O. Anderson, S. Dasgupta, P. Khargonekar, F. J. Kraus and M. Mansour. Robust Strict Positive Realness: Characterization and Construction. IEEE Transactions on Circuits and Systems, Vol. 37, No. 7, pp. 869-876, 1990.

[Åström, 2000] K. J. Åström. Limitations on Control System Performance. European Journal of Control, Vol. 6, No. 1, pp. 2-20, 2000.

[Ben-Tal, 2000] A. Ben-Tal, L. El Ghaoui and A. Nemirovski. Robust Semidefinite Programming. In R. Saigal, L. Vandenberghe and H. Wolkowicz (Editors). Handbook of Semidefinite Programming. Kluwer Academic Publishers, Boston, Massachusetts, 2000 .

[Bhattacharyya, 1995] S. P. Bhattacharyya, H. Chapellat and L. H. Keel. Robust Control: The Parametric Approach. Prentice Hall, Upper Saddle River, New Jersey, 1995.

[Blondel, 2000] V. D. Blondel and J. N. Tsitsiklis. A Survey of Computational Complexity Results in Systems and Control. Automatica, Vol. 36, pp. 1249-1274, 2000.

[Braatz, 1998] R. D. Braatz and O. D. Crisalle. Robustness Analysis for Systems with Ellipsoidal Uncertainty. International Journal of Robust and Nonlinear Control, Vol. 8, pp. 1113-1117, 1998.

[Chilali, 1996] M. Chilali and P. Gahinet. H-infinity Design with Pole Placement Constraints: An LMI Approach. IEEE Transactions on Automatic Control, Vol. 41, No. 3, pp. 358-367, 1996.

[Dorato, 2000] P. Dorato. Analytic Feedback System Design: An Interpolation Approach. Brooks Cole Publishing, New York, 2000.

[Doyle, 1992] J. C. Doyle, B. A. Francis and A. R. Tannenbaum. Feedback Control Theory. MacMillan, New York, 1992.

[Dumitrescu, 2002] B. Dumitrescu. Parameterization of positive-real transfer functions with fixed poles. IEEE Transactions on Circuits and Systems I: Fundamental Theory and Applications, Vol. 49, No. 4, pp. 523-526, 2002. 
[El Ghaoui, 1999] L. El Ghaoui, S. I. Niculescu (Editors). Advances in Linear Matrix Inequality Methods in Control. SIAM, Philadelphia, 1999.

[Genin, 2002] Y. Genin, Y. Hachez, Yu. Nesterov, R. Ştefan, P. Van Dooren and S. Xu. Positivity and Linear Matrix Inequalities. European Journal of Control, Vol. 8, pp. 275-298, 2002.

[Henrion, 2001a] D. Henrion and O. Bachelier. Low-Order Robust Controller Synthesis for Interval Plants. International Journal of Control, Vol. 74, No. 1, pp. 1-9, 2001.

[Henrion, 2001b] D. Henrion, D. Arzelier, D. Peaucelle and M. Šebek. An LMI Condition for Robust Stability of Polynomial Matrix Polytopes. Automatica, Vol. 37, No. 3, pp. 461-468, 2001.

[Henrion, 2001c] D. Henrion, O. Bachelier, M. Šebek. D-Stability of Polynomial Matrices. International Journal of Control, Vol. 74, No. 8, pp. 845-856, 2001.

[Kučera, 1979] V. Kučera. Discrete Linear Control: The Polynomial Approach. John Wiley and Sons, Chichester, 1979.

[Langer, 1999] J. Langer, I. D. Landau. Combined Pole Placement/Sensitivity Function Shaping Method using Convex Optimization Criteria. Automatica, Vol. 35, No. 6, pp. 1111-1120, 1999.

[Lasserre, 2001] J. B. Lasserre. Global Optimization with Polynomials and the Problem of Moments. SIAM Journal on Optimization, Vol. 11, No. 3, pp. 796-817, 2001.

[Nesterov, 2000] Yu. Nesterov. Squared Functional Systems and Optimization Problems. Chapter 17, pp. 405-440 in H. Frenk, K. Roos, T. Terlaky and S. Zhang (Editors). High Performance Optimization. Kluwer Academic Publishers, Dordrecht, The Netherlands, 2000 .

[Parrilo, 2000] P. A. Parrilo. Structured Semidefinite Programs and Semialgebraic Geometry Methods in Robustness and Optimization. PhD Thesis, California Institute of Technology, Pasadena, California, 2000.

[Peaucelle, 2000] D. Peaucelle, D. Arzelier, O. Bachelier, J. Bernussou. A New Robust D-Stability Condition for Real Convex Polytopic Uncertainty. Systems and Control Letters, Vol. 40, pp. 21-30, 2000.

[Polyx Ltd., 2001] Polyx, Ltd. The Polynomial Toolbox for Matlab. Version 2.5 released in 2001. Version 3.0 to be released soon. See www.polyx.cz.

[Rantzer, 1994] A. Rantzer and A. Megretski. A Convex Parameterization of Robustly Stabilizing Controllers. IEEE Transactions on Automatic Control, Vol. 39, No. 9, pp. 1802-1808, 1994.

[Skelton, 1998] R. E. Skelton, T. Iwasaki and K. Grigoriadis. A Unified Algebraic Approach to Linear Control Design. Taylor and Francis, London, 1998.

[Stipanović, 2000] D. M. Stipanović and D. D. Šiljak. Robust Strict Positive Realness via Polynomial Positivity.Proceedings of the American Control Conference, Chicago, Illinois, pp. 4318-4325, 2000. 
[Sturm, 1999] J. F. Sturm. Using SeDuMi 1.02, a Matlab Toolbox for Optimization over Symmetric Cones. Optimization Methods and Software, Vol. 11-12, pp. 625-653, 1999. See also fewcal.kub.nl/sturm.

[Tong, 1994] Y. Tong and N. K. Sinha. A Computational Technique for the Robust Root Locus. IEEE Transactions on Industrial Electronics, Vol. 41, No. 1, pp. 79-85, 1994.

[Vandenberghe, 1996] L. Vandenberghe and S. P. Boyd. Semidefinite Programming. SIAM Review, Vol. 38, No. 1, pp. 49-95, 1996.

[Vidyasagar, 1985] M. Vidyasagar. Control System Synthesis. A Factorization Approach. The MIT Press, Cambridge, Massachusetts, 1985.

[Wang, 2000] S. Wang and J. H. Chow. Low-Order Controller Design for SISO Systems Using Coprime Factors and LMI. IEEE Transactions on Automatic Control, Vol. 45, No. 6, pp. 1166-1169, 2000.

[Zhou, 1996] K. Zhou, J. C. Doyle and K. Glover. Robust and Optimal Control. Prentice Hall, Upper Saddle River, New Jersey, 1996. 